\title{
ELECTRIC POLARIZATION OF ONSAGER FLUIDS II. BIREFRINGENCE. 1. KERR CONSTANTS OF PURE SUBSTANCES
}

\author{
V.V. Prezhdo ${ }^{a}$, G.V. Tarasova ${ }^{b}$, O.V.. Prezhdo ${ }^{c}$, S.A. Tyurin ${ }^{b}$, \\ O.N. AKULOVA ${ }^{b}$ AND T.N. KURSKaYA ${ }^{b}$ \\ a Institute of Chemistry, Pedagogical University, Oleska 48, 45-052 Opole, Poland \\ ${ }^{b}$ Ukrainian Scient. Center for Water Control, Bakunina 6, Kharkow 310001, Ukraine

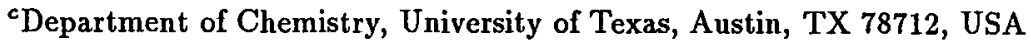

(Received October 20, 1993)

The Kerr constants of pure liquid organic compounds, which exhibit weak intermolecular interactions and possess widely varying electric, optical and polarizational molecular parameters, were computed from the data on dielectrometry and electric birefringence. Good agreement of the calculated and observed values was reached as the result of the introduction of the local field model, which develops the concepts of reactive field and local electric induction of a polarized fluid, into the orientational theory of the Kerr effect.

PACS numbers: $78.20 . \mathrm{Fm}, \mathbf{7 2 . 8 0 . J c}$

\section{Introduction}

The Kerr effect - electric birefringence (EB) - dwells among the most sensible and precise methods of investigation of linear and nonlinear optical and electric properties of molecules. Unambiguous interpretation of the effect requires a theory that relates composition and structure of a substance with its electro-optical parameters. Langevin-Born's orientational theory is undoubtedly adequate for gaseous media. Molecular-statistic description of the Kerr effect based on molecular correlations assumes cognizance of a good deal of empirical and calculated values and therefore is applicable to a small number of well studied substances. It is the reason that induces researchers to extend the orientational theory of EB to condensed media through the introduction of local fields. Analysis of published results of theoretical approaches of this kind witnesses a substantial disparity between theory and experiment that is attributable to underdeveloped physical foundations of models of interrelation between Maxwell's and local fields in anisotropic polarized molecular fluids.

The paper presents the outcomes of theoretical description of the Kerr effect in liquids according to Langevin-Born employing the local field model that we have 
worked out in the previous publications [1]. Experimental part of it comprises the comparison of the EB constants computed by the expressions derived with the observed ones measured for liquid organic media.

\section{Theoretical description of the Kerr effect in liquids}

Total energy of unit volume, that contains $N$ molecules, of an electrically polarized medium is $U_{\boldsymbol{\Sigma}} \approx \boldsymbol{P}_{\boldsymbol{\Sigma}} \boldsymbol{E}$, where polarization $\boldsymbol{P}_{\boldsymbol{\Sigma}}$ in the absence of intermolecular interaction constitutes the sum of orientation $\left(\boldsymbol{P}_{\mathrm{or}}=\sum_{i} \boldsymbol{P}_{\mathrm{or}}\right)$ and electronic $\left(\boldsymbol{P}_{\mathrm{el}}=\sum_{i} \boldsymbol{P}_{\mathrm{el}_{i}}\right)$ polarizations originated by the local field $\boldsymbol{E}_{l}$ of the fluid placed in the external electric field $\boldsymbol{E}$. We have deduced earlier the equations for $\boldsymbol{E}_{l}$ [2] within the limits of the model evolving the concepts of reactive field of a molecular polarizable spherical body (with a rigid dipole in the center) and local distribution of electric induction of its nearest surroundings. Thus, the components of the effective molecular moment of the medium polarized by the field $\boldsymbol{E}_{l}$ along the directions of the dipole $\boldsymbol{\mu}_{0}^{*}$ and the external field $\boldsymbol{E}(\xi-$ instantaneous angle of their mutual orientation) have the appearance

$$
\begin{aligned}
& m_{\mu}=(1+\alpha t) \mu_{0}^{*}+\alpha G^{*}\langle\cos \xi\rangle, \\
& m_{E}=\alpha G^{*}+\alpha R_{0}^{*}\langle\cos \xi\rangle, \\
& G^{*}=g E(1-\alpha t)^{-1}, \quad R_{0}^{*}=t \mu_{0}^{*}=t \mu_{0}(1-\alpha t)^{-1}, \\
& \langle\cos \xi\rangle=(1+1.5 \alpha t) \mu_{0}^{*} G^{*} / 3 k T,
\end{aligned}
$$

where $\alpha$ - average dynamic polarization of molecules; $g\left(\varepsilon_{0}, \varepsilon_{\infty}\right), t\left(\varepsilon_{0}, \varepsilon_{\infty}, \alpha\right)-$ cavity $G^{*}$ and reactive $R_{0}^{*}$ field factors $[3,4] ; \varepsilon_{0}, \varepsilon_{\infty}$ - low- and high-frequency permittivities of a medium; $a$ - molecular cavity radius calculated according to [5]; $\mu_{0}$ - electric moment of a free molecule. Consequently, the introduced above molecular polarizations are

$$
P_{\mathrm{el}}=m_{E}, \quad P_{\mathrm{or}}=m_{\mu}\langle\cos \xi\rangle .
$$

Statistic distribution of anisotropic polar molecules of a fluid in various independent directions in the external field $E$ is described by Boltzmann's function $f \approx \exp \left(-U_{\Sigma} / k T\right)$. To define its explicit form let us introduce autonomous coordinate systems: the laboratory one $\{\sigma\}=(x, y, z)$, with the axis $z$ along the field $E$, and molecular coordinate systems $\{i\}=(1,2,3)$ specified by the main axes of molecular ellipsoids of polarizability. Mutual orientations of $\{\sigma\}$ and $\{i\}$ are determined by guiding cosines $\cos [i, \sigma] \equiv(i, \sigma)$, related to Euler's angles $\{\tau\}=(\phi, \psi, \theta)$ by known expressions. Then, molecular polarization contributions along the external field can be rewritten according to (1) as

$$
\begin{aligned}
& P_{\mathrm{el}_{z}}=\sum_{i} P_{\mathrm{el}_{i}}=\sum_{i} m_{E_{i}}(i z), \\
& P_{\mathrm{or}_{z}}=\sum P_{\mathrm{or}_{i}}=G^{*}(1+1.5 \alpha t) \sum_{i, j} m_{\mu_{i}} \mu_{0_{j}}^{*}(i z)(j z) / 3 k T .
\end{aligned}
$$

Once the instantaneous energies of polarized molecular moments in the field $E$ are calculated, expanding Boltzmann's distribution function $\exp \left(-U_{\Sigma} / k T\right)$ in terms 
of the small parameter $U_{\Sigma} / k T \ll 1$ and truncating the series after the quadratic term, we obtain

$$
\begin{aligned}
& f(u, \tau)=A\left[1+\frac{E^{2}}{2 k T} g^{*} \sum_{i}\left(\alpha_{i}^{e}+(1+1.5 \alpha t)^{2} \frac{\mu_{0_{i}}^{*}}{k T}\right)(i z)^{2}\right. \\
& \left.+\frac{E^{2}}{2 k^{2} T^{2}} g^{*}(1+1.5 \alpha t)^{2} \sum_{i, j} \mu_{0_{i}}^{*} \mu_{0_{j}}^{*}(i z)(j z)+\ldots\right]
\end{aligned}
$$

where the constant $A$ is inferred from the normalization criterion $\int f(u, \tau) \mathrm{d} \tau$, $\mathrm{d} \tau=\mathrm{d} \phi \mathrm{d} \psi \sin \theta \mathrm{d} \theta ; \alpha_{i}^{e}$ are the components of the transformed to major axes centrosymmetric hermitian, 2nd-rank tensor $A_{i j}^{e}$ of low-frequency (statistic) polarizability of molecules

$$
\alpha_{i}^{e}=\sum_{j} \alpha_{j}^{e}(j z)^{2}, \quad \mu_{0_{i}}^{*}=\sum_{j} \mu_{0_{j}}^{*}(j z) .
$$

By virtue of the theorem on the averaged value of a function of cosines $\overline{[[(i z)]}=$ $\left(8 \pi^{2}\right)^{-1} \iiint_{0}^{2 \pi} \int f[(i z)] \mathrm{d} \tau$, and consequently, $\overline{(i j)(k n)}=\delta_{i k} \delta_{j n} / 3, \delta_{m p}-$ Kronecker symbol) upon integration we achieve

$$
\text { - } \frac{1}{A}=8 \pi^{2}\left[1+\frac{E^{2}}{6 k T} g^{*} \sum_{i}\left(\alpha_{i}^{e}+(1+1.5 \alpha t)^{2} \frac{\mu_{0_{i}}^{*^{2}}}{k T}\right)+\ldots\right] .
$$

And, finally, with regard to infinitesimal in comparison with unity magnitudes of the second summand in (3) substituting the expression for $A$ into (2) we can represent Boltzmann's distribution of anisotropic molecules of an electrically polarized fluid in the form

$$
\begin{array}{r}
f(u, \tau)=\frac{1}{8 \pi^{2}}\left[1-\frac{E^{2}}{6 k T} g^{*} \sum_{i}\left(\alpha_{i}^{e}+(1+1.5 \alpha t)^{2} \frac{\mu_{0_{i}}^{*^{2}}}{k T}\right)\right. \\
\left.+\frac{E^{2}}{2 k T} g^{*} \sum_{i}\left(\alpha_{i}^{e}+(1+1.5 \alpha t)^{2} \frac{\mu_{0_{i}}^{*^{2}}}{k T}\right)(i z)^{2}+\ldots\right] .
\end{array}
$$

When deducing the formulas $(3,4)$ we omit the terms with the factors of the types $(i z)^{2 k+1}$ and $(i z)^{2 k+1}(j z)^{2 k+1}, k$ and $l=0,1,2 \ldots$

Optical (high-frequency) polarizability of molecules of conservative systems, that governs, in particular, optical properties of a fluid polarized by the field $E$, is also described by a hermitian 2nd-rank tensor $A_{i, j}^{0}$ with the components depending, in general, upon the field

$$
\begin{aligned}
& A_{i j}^{0}=\alpha_{i j}^{0}+\sum_{k} \alpha_{i j, k}^{0} E_{k}+\frac{1}{2} \sum_{k, l} \alpha_{i j, k l}^{0} E_{k} E_{l}+\ldots \\
& \alpha_{i j, k}^{0}=\left(\partial A_{i j}^{0} / \partial E_{k}\right)_{E=0} \text { and } \alpha_{i j, k l}^{0}=\left(\partial^{2} A_{i j}^{0} / \partial E_{k} \partial E_{l}\right)_{E=0} .
\end{aligned}
$$

Now we find the statistically averaged value of $A_{i j}^{0}$ in the laboratory coordinate system $\{\sigma\}: \overline{A_{i j}^{0}}=\int f(u, \tau) A_{i j}^{0}(i \sigma)(j \gamma) \mathrm{d} \tau$. The second and the third summands in (5) are hermitian (indices $i, j$ ) tensors of 3rd- and 4th-ranks and are bound up with the Stark and secondary Stark effects. They represent the Voigt contributions to the anisotropy of optical polarizability of a medium. By virtue of the negligibility 
of these terms (away from absorption lines Voigt contributions to the forced optical anisotropy of a medium are three orders less than orientational ones) they can be left out. Specifying the external field $E=E_{z}=E(k z)$, and substituting the distribution function (4) into the expression for $\overline{A_{i j}^{0}}$ with an accuracy to the second order of $E^{2}$ we get

$$
\begin{aligned}
\overline{A_{\sigma \gamma}^{0}} & =\frac{1}{8 \pi^{2}} \int\left[\sum_{i, j} \alpha_{i j}^{0}(i \sigma)(j \gamma)\right. \\
& -g^{*} \frac{E^{2}}{6 k T} \sum_{i, j, k} \alpha_{i j}^{0}\left(\alpha_{k}^{e}+(1+1.5 \alpha t)^{2} \frac{\mu_{0_{k}}^{*^{2}}}{k T}\right)(i \sigma)(j \gamma) \\
& \left.+g^{*} \frac{E^{2}}{2 k T} \sum_{i, j, k} \alpha_{i j}^{0}\left(\alpha_{k}^{e}+(1+1.5 \alpha t)^{2} \frac{\mu_{0_{k}}^{*^{2}}}{k T}\right)(i \sigma)(j \gamma)(k z)^{2}\right] \mathrm{d} \tau .
\end{aligned}
$$

Going in (6) from integration to averaging, accounting for the following equation for a taken spatial direction (axis $z$ ):

$$
\begin{aligned}
& \overline{(k i)(n j)(l z)(p z)}=\xi \delta_{k n} \delta_{l p}+\gamma\left(\delta_{k n} \delta_{l p}+\delta_{k p} \delta_{n l}\right), \\
& \xi=(1 / 15)\left(2 \delta_{i j}-\delta_{i z} \delta_{j z}\right), \quad \gamma=(1 / 30)\left(3 \delta_{i z} \delta_{j z}-\delta_{i j}\right),
\end{aligned}
$$

and assuming that the major axes $(1,2,3)$ of ellipsoids of high- and low-frequency molecular polarizabilities coincide (that holds for conservative systems), we arrive at

$$
\begin{aligned}
& \overline{A_{y y}^{0}}=\overline{A_{x x}^{0}}=\overline{\alpha^{0}}-g^{*} \frac{E^{2}}{90 k T}\left\{\left(\alpha_{1}^{0}-\alpha_{2}^{0}\right)\left(\alpha_{1}^{e}-\alpha_{2}^{e}\right)\right. \\
&+\left(\alpha_{1}^{0}-\alpha_{3}^{0}\right)\left(\alpha_{1}^{e}-\alpha_{3}^{e}\right)+\left(\alpha_{2}^{0}-\alpha_{3}^{0}\right)\left(\alpha_{2}^{e}-\alpha_{3}^{e}\right) \\
&-\frac{(1+1.5 \alpha t)^{2}}{k T}\left[\left(\alpha_{1}^{0}-\alpha_{2}^{0}\right)\left(\mu_{0_{1}}^{*^{2}}-\mu_{0_{2}}^{*^{2}}\right)+\left(\alpha_{1}^{0}-\alpha_{3}^{0}\right)\left(\mu_{0_{1}}^{*^{2}}-\mu_{0_{3}}^{*^{2}}\right)\right. \\
&\left.\left.+\left(\alpha_{2}^{0}-\alpha_{3}^{0}\right)\left(\mu_{0_{2}}^{*^{2}}-\mu_{0_{3}}^{*^{2}}\right)\right]\right\}=\overline{\alpha^{0}}-\frac{g}{2(1-\alpha t)}\left[\Theta_{1}^{g}+\left(\frac{1+1.5 \alpha t}{1-\alpha t}\right)^{2} \Theta_{2}^{g}\right] E^{2}, \\
& \overline{A_{z z}^{0}}=\overline{\alpha^{0}}+\frac{g}{1-\alpha t}\left[\Theta_{1}^{g}+\left(\frac{1+1.5 \alpha t}{1-\alpha t}\right)^{2} \Theta_{2}^{g}\right] E^{2}, \\
& \overline{A_{x y}^{0}}=\overline{A_{x z}^{0}}=0, \quad \overline{\alpha^{0}}=\left(\alpha_{1}^{0}+\alpha_{2}^{0}+\alpha_{3}^{0}\right) / 3,
\end{aligned}
$$

$\Theta_{1}^{g}$ and $\Theta_{2}^{g}$ - anisotropic and dipole contributions according to Langevin-Born's theory of EB. It follows that induced optical anisotropy of a molecular medium affected by the external electric field $E=E_{z}$ in the $(y z)$ plane perpendicular to a beam of light (axis $x$ of the $\{\sigma\}$ coordinate system) equals

$$
\overline{A_{z z}^{0}}-\overline{A_{y y}^{0}}=\alpha_{\|}^{0}-\alpha_{\perp}^{0}=(3 / 2) \frac{g}{1-\alpha t}\left[\Theta_{1}^{g}+\left(\frac{1+1.5 \alpha t}{1-\alpha t}\right)^{2} \Theta_{2}^{g}\right] \text {. }
$$

As ascertained by experiments, for conservative systems and for identical orientations of the major axes of $A_{i j}^{0}$ and $A_{k l}^{e}$ within several percents the following relationship takes place [6]:

$$
\frac{\overline{\alpha^{e}}}{\overline{\alpha^{0}}}=\frac{\alpha_{i j}^{e}}{\alpha_{k l}^{0}}=A=\left\{\begin{array}{cc}
\left(n_{\infty}^{2}-1\right)\left(n^{2}-1\right)^{-1}, & \text { when } \mu_{0} \neq 0 \\
\left(\varepsilon_{0}^{2}-1\right)\left(n^{2}-1\right)^{-1}, & \text { when } \mu_{0}=0
\end{array}\right.
$$


For slightly polar fluids $\left(\varepsilon_{0}<10\right) A \approx 1$, and for $10<\varepsilon_{0}<20$, $A \approx 1.05-1.10 ; n_{\infty}^{2}$ is the refraction index of a medium, extrapolated to infinitely long waves. That is why in the general case of a non-diagonalized tensor $A_{i j}^{0}$ we have

$$
\Theta_{1}^{g}=\frac{A}{45 k T}\left(2 \sum_{i, j} \alpha_{i}^{0} \alpha_{j}^{0}-6 \sum_{i, j}^{\prime} \alpha_{i}^{0} \alpha_{j}^{0}\right)=\frac{2 A}{45 k T} \gamma^{2},
$$

$\gamma^{2}-$ optical anisotropy.

Interconnection between the optical polarizability of molecules and the refraction index of a medium is established by the famous Lorentz-Lorenz' expression. The later presumes isotropy of the internal field of a wave of light given in the form of Lorenz' field $\left(E_{\mathrm{L}}=E\left(n^{2}+2\right) / 3\right)$. However, any optically isotropic medium placed in the polarizing electric field gains the properties of an one-axis crystal. The problem of adaptation of the expression cited above to anisotropic media was widely discussed in literature and found its solution in the experimental studies on optical polarization of crystals conducted by Vuks [7]: in an anisotropic substance acting electric field of a wave of light (Lorenz' field) remains practically isotropic. Ergo

$$
3\left(n_{E_{i}}{ }^{2}-1\right)=4 \pi N_{E_{i}} \alpha_{E_{i}}\left(n^{2}+2\right),
$$

$n_{E_{i}}$ is the refraction index of a polarized by the electric field $E$ substance in the $i$-th direction $\left(E: n_{E}=n\right.$ and $\left.\perp E: n_{\perp}\right) ; n^{2}=\overline{n^{2} E_{i}}=\left(n^{2}+2 n_{\perp}^{2}\right) / 3=n_{E=0}^{2}$; $N_{E_{i}}=N\left[1+\left(\partial \varepsilon_{0} / \partial p\right)-E^{2} / 8 \pi\right]$ is the number of particles in a unit volume of a medium, the number is conditioned by electrostriction in the $i$-th direction; $p$ - pressure. After logarithmic differentiation and termwise division by Lorentz-Lorenz' expression we obtain

$$
\Delta n=n-n_{\perp}=2 \pi N\left(\alpha^{0}-\alpha_{\perp}^{0}\right)\left(n^{2}+2\right) / 3 n .
$$

$\alpha^{0}$ and $\alpha_{\perp}^{0}$ are optical polarizabilities of molecules in the directions parallel and perpendicular to the external electric field vector $\boldsymbol{E}$.

Keeping with the empirical Kerr law $K=\lim \left(\Delta n / n E^{2}\right), E \rightarrow 0$ and employing $(8,10)$ we get the following expression for the EB constant of a molecular fluid:

$$
\begin{aligned}
K^{l} & =\pi N\left(n^{2}+2\right) g^{*}\left[\Theta_{1}^{g}+(1+1.5 \alpha t)^{2} \Theta_{2}^{g}(1-\alpha t)^{-2}\right] n^{-2} \\
& =\pi N\left(n^{2}+2\right) g^{*}\left[\Theta_{1}^{g}+\Theta_{2}^{l}\right] n^{-2}=\pi N C_{\mathrm{L}}^{0} C_{\mathrm{A}}^{e}\left(\Theta_{1}^{g}+\Theta_{2}^{l}\right),
\end{aligned}
$$

where $C^{0, e}=E_{e}^{0, e} / E^{0, e}$ are determined by the type of the model description of the relationship between Maxwell's and local (high- and low-frequency) fields in a polarized fluid: by Lorenz $-C_{\mathrm{L}}^{0}$ and by $[3,4]-C_{\mathrm{A}}^{e}$. The equation developed (11) differs from the corresponding formulas of the orientational [8] and phenomenological $[9,10]$ theories not only in the first powers of $C^{0, e}$, but also in the shape of the dipole term $\Theta_{2}^{l}$. 


\section{Results and discussion}

To experimentally test the expression (11) and to compare it with the results of known theories of EB of liquids we have measured the Kerr constants $B_{e}^{l}=n K_{e}^{l} \lambda^{-1}(\lambda-$ wavelength of the analyzing beam of light that goes through a medium) for a series of liquid organic compounds with weak intermolecular interaction and of a wide range of molecular polarities, polarizabilities and their anisotropies. The experimental equipment and the procedure of compensational measurements are detailed in [11].

TABLE

Comparison of experimental and calculated values of the Kerr constants of nonpolar (1-8) and polar (9-15) liquid organic compounds.

\begin{tabular}{r|l|c|r|c|c|c|c|c}
\hline \hline No. & Substance & $\begin{array}{c}B_{\mathrm{e}}^{l} \cdot 10^{-8} \\
\text { CGSE }\end{array}$ & $\Delta B_{1}^{l}$ & $\Delta B_{2}^{l}$ & $\Delta B_{3}^{l}$ & $\Delta B_{4}^{l}$ & $\Delta B_{5}^{l}$ & $\Delta B_{6}^{l}$ \\
\hline 1 & $\mathrm{C}_{8} \mathrm{H}_{10}$ & 6.800 & 1.513 & 1.474 & 0.818 & 0.191 & 0.172 & 0.254 \\
2 & $\mathrm{C}_{9} \mathrm{H}_{12}$ & 6.211 & 1.154 & 1.446 & 0.776 & 0.182 & 0.169 & 0.067 \\
3 & $\mathrm{C}_{6} \mathrm{H}_{6}$ & 3.552 & 1.529 & 1.456 & 0.803 & 0.182 & 0.169 & 0.254 \\
4 & $\mathrm{CH}_{3}\left(\mathrm{CH}_{2}\right)_{10} \mathrm{CH}_{3}$ & 1.106 & 0.448 & 0.383 & 0.199 & 0.127 & 0.128 & 0.191 \\
5 & $\mathrm{CH}_{3}\left(\mathrm{CH}_{2}\right)_{6} \mathrm{CH}_{3}$ & 0.713 & 0.234 & 0.174 & 0.128 & 0.107 & 0.084 & 0.297 \\
6 & $\mathrm{CH}_{3}\left(\mathrm{CH}_{2}\right)_{5} \mathrm{CH}_{3}$ & 0.631 & 0.165 & 0.148 & 0.119 & 0.089 & 0.074 & 0.317 \\
7 & $\mathrm{CH}_{3}\left(\mathrm{CH}_{2}\right)_{4} \mathrm{CH}_{3}$ & 0.499 & 0.169 & 0.142 & 0.101 & 0.076 & 0.066 & 0.397 \\
8 & $\mathrm{CH}_{3}\left(\mathrm{CH}_{2}\right)_{3} \mathrm{CH}_{3}$ & 0.452 & 0.011 & 0.008 & 0.006 & 0.004 & 0.004 & 0.285 \\
9 & $\mathrm{CH}_{3} \mathrm{CN}_{10}$ & 481.48 & 39.886 & 12.17 & 5.129 & 1.168 & 0.754 & 2.629 \\
11 & $\mathrm{C}_{5} \mathrm{H}_{5} \mathrm{~N}$ & 198.23 & 4.817 & 1.973 & 0.426 & 0.212 & 0.109 & 0.189 \\
12 & $\left.\mathrm{CH}_{3}\right)_{2} \mathrm{CHOH}$ & 193.23 & 10.544 & 4.715 & 1.621 & 0.673 & 0.288 & 0.167 \\
13 & $\mathrm{C}_{6} \mathrm{H}_{5} \mathrm{Cl}$ & 110.84 & 2.257 & 1.017 & 0.725 & 0.362 & 0.194 & 0.116 \\
14 & $\mathrm{C}_{2} \mathrm{O}$ & 38.00 & 15.300 & 6.524 & 2.363 & 0.873 & 0.317 & 0.520 \\
15 & $\mathrm{CHCl}_{3}$ & 7.26 & 0.409 & 0.371 & 0.206 & 0.113 & 0.096 & 0.315 \\
\hline
\end{tabular}

1. 1,4-xylene; 2 . mesitylene; 3 . benzene; 4. dodecane; 5 . octane; 6 . heptane; 7. hexane; 8. pentane; 9 . acetonitrile; 10. pyridine; 11. 2-propanol; 12. chlorobenzene; 13 . water; 14 . toluene; 15 . chloroform.

The data presented in Table on the experimental and calculated (theoretical) values of EB constants $\left(\Delta B_{i}^{l}=\left|B_{e}^{l}-B_{t}^{l}\right|\left(B_{e}^{l}\right)^{-1}\right)$ have been obtained within the limits of the orientational theory with Lorenz' local field $(i=1)$, Onsager's one $(i=2)$, Block-Walker's one [12] $(i=3)$, and our approach (11) with the factors $C_{\mathrm{A}}^{e}$ defined in [3] $(i=4)$ and [4] $(i=5)$. The results of computations by Vuks' phenomenological theory [10] $(i=6)$ are displayed there as well. 
In compliance with Langevin-Born's approximation, for EB constants of apolar substances holds $B \approx \gamma^{2}$, which is true for our data (compounds 1-8): the magnitudes of optical anisotropies of $\mathrm{C}_{8} \mathrm{H}_{10}$ and $\mathrm{CH}_{3}\left(\mathrm{CH}_{2}\right)_{3} \mathrm{CH}_{3}$ are 78.60 and $3.12 \AA^{6}$. More complex relationship between the constant $B^{l}$ and molecular parameters is typical of polar substances due to constant's additivity over orientational $\left(\Theta_{1}>0\right)$ and dipole $\left(\Theta_{2} \neq 0\right)$ terms in (11). Generally, the correlation between EB constants and molecular moments (compounds 9-15) takes place: $\mu_{0}$ of $\mathrm{C}_{7} \mathrm{H}_{8}$ and $\mathrm{CH}_{3} \mathrm{CN}$ equal to 0.37 and $3.97 \mathrm{D}$ correspondingly. Nevertheless, the Kerr constants of substaners with similar molecular dipoles can vary considerably not only in absolute values but also in signs. For instance, for chlorobenzene $\left(\mu_{0}=1.72 \mathrm{D}\right.$, $\left.\gamma^{2}=65.2 \AA^{6}\right) B^{l}>0$, while for trichloromethane $\left(\mu_{0}=1.86 \mathrm{D}, \gamma^{2}=9.27 \AA^{6}\right)$ $B^{l}<0$, since the later possesses $\Theta_{2}^{l}<0, \Theta_{l}^{g}<\left|\Theta_{1}^{l}\right|$.

The data in Table reflect the actual influence of intermolecular interactions, which are not accounted for by the orientational theory, on the electro-optical characteristics of a fluid. In this connection we wish to note straight correlation between the magnitudes of relative errors in the Kerr constants $\left(\Delta B_{i}^{l}\right)$, molecular moments and polarizabilities (and their anisotropies). Thus, $\Delta B_{i}^{l}$ of $\mathrm{C}_{8} \mathrm{H}_{10}$ is greater than that of $\mathrm{CI}_{3}\left(\mathrm{CH}_{2}\right)_{3} \mathrm{CH}_{3}$, as well as $\Delta B_{i}^{l}$ of acetonitrile is the largest for all polar fluids investigated.

The mentioned above discrepancy between the calculated in the context of Langevin-Born's theory and experimental values of the Kerr constants is chiefly conditioned by the types of model description of both relationship between Maxwell's and local fields and anisotropy of molecular polarizability. Incorporation of the Onsager internal field, that implicitly (via the reactive field) allows for intermolecular interactions somewhat improves computed birefringence constants: $\Delta B_{2}^{l}<\Delta B_{1}^{l}$ for all pure organic liquids examined. Similar tendency is revealed in the event of the introduction of the model description of dielectric permittivity $\varepsilon_{0}$ at the boundary "Onsager cavity-continuum" by a fair function [12]: $\Delta B_{2}^{l}>\Delta B_{3}^{l}$. Lastly, the findings of a consistent analysis of inductive-orientational contributions of the reactive field into the potential energies of particles of an electrically polarized fluid, a comparison of gaseous and liquid phase values of molecular moments, and an adoption of the local molecular distribution of electric induction of a medium as a function of distance from a fixed particle: either reverse exponent $(i=4)$ or oscillatory quickly quenching one related to the radial function of arrangement of molecules in a fluid $(i=5)$ evidence that: $\Delta B_{3}^{l}>\Delta B_{4}^{l}>\Delta B_{5}^{l}$. Juxtaposition of the errors $\Delta B_{i}^{l}$ for nonpolar and polar compounds can also lend credence to the fact that the enumerated above modified descriptions of the internal field and electric polarization of a fluid implicitly account for intermolecular interactions. The results of various theoretical approaches to the calculation of EB constants of molecular fluids with weak interparticle coupling, in line with the actual data presented here, display the following tendency:

$\Delta B_{1}^{l}>\Delta B_{2}^{l}>\Delta B_{3}^{l}>\Delta B_{6}^{l}>\Delta B_{4}^{l} \geq \Delta B_{5}^{l}$.

The outcomes of the conducted in this paper calculations of the Kerr constants of some pure organic liquids and their deviations from the values observed clearly demonstrate far from being exhausted resources of Langevin-Born's theory as to its enhancement via the development of the local field concept. 


\section{References}

[1] V.V. Prezhdo, G.V. Tarasova, O.V. Prezhdo, S.A. Tyurin, O.N. Akulova, T.N. Kurskaya, Acta Phys. Pol. A 84, 253 (1993).

[2] G.V. Tarasova, Ph.D. Thesis, Kharkov University, Klarkov 1991, p. 25.

[3] S.A. Tyurin, M.V. Hastchina, Yu.D. Feldman, Acta Phys. Pol. A 70, 696 (1986).

[4] S.A. Tyurin, M.V. Hastchina, Zh. Fiz. Khim. (USSR) 62, 1680 (1987).

[5] B.A. Gorodyssky, N.G. Bakhshiev, L.F. Kardashina, Spektr. Vnutri- Mezhmolek. Vzaimod. (USSR) 1, 63 (1975).

[6] A.N. Vereshchagin, Polarizability of Molecules, Nauka, Moscow 1980, p. 177.

[7] M.F. Vuks, N.B. Rozhdestvenskaya, K. Eydner, Opt. Spektrosk. (USSR) 45, 114 (1978).

[8] S. Filipczuk, G. Khanarian, J. Chem. Soc. Faraday Trans. 77, 477 (1981).

[9] V.A. Zamkov, in: Optical Investigations of Liquids and Solutions, Nauka, Tashkent: Fan 1965, p. 128.

[10] V.A. Vuks, Opt. Spektrosk. (USSR) 21, 667 (1966).

[11] V.V. Prezhdo, M.V. Hastchina, V.A. Zamkov, Electro-optical Investigations in Physics and Chemistry, Vyssha Shkola (KSU), Kharkov 1982, p. 152.

[12] H. Block, Z.M. Walker, Chem. Phys. Lett. 19, 363 (1973). 